\title{
Torque and Current Ripple Reduction in PM Generator based Multi Modular - Multi MW WECSs with Extended PWM Inverter Modulation
}

\author{
Antonino Di Gerlando, Khaled ElShawarby, Giovanni Maria Foglia, Roberto Perini
}

\begin{abstract}
The paper presents a method to reduce both the torque ripple and the dc current ripple in the machine side converter, in a multi megawatt Wind Energy Conversion System, based on a PM Synchronous Generator which has a multi modular structure. Each converter module consists of a three-phase, two level inverter, operating as a rectifier, with PWM sinusoidal modulation. The first aim of the method is exploiting not only the linear PWM region but also over modulation region, in order to allow a higher $\mathrm{AC}$ voltage, with the same de link voltage. The ripple mitigation is gained by means of a suited shifting among the carrier signals and the control signals in the several modules. The paper describes the method, and discusses the criteria that can be adopted to choose the suited shifting angles. Some simulation results are reported.
\end{abstract}

Index Terms -- Permanent Magnet Synchronous Machines, Modular Machines, inverter, torque ripple, current ripple.

\section{INTRODUCTION}

I $\mathrm{n}$ large capacity power systems (multi megawatt), multi modular and/or multiphase electrical machines have been gaining rising interest, due to several features:

- higher reliability: if one part of the system fails, the others can be kept in operation;

- total power sharing among several parts of reduced ratings;

- reduction of the ripples in the electromechanical quantities (currents and torques), by means of suited shifting among the electrical quantities in the several modules.

Actually, these remarks hold also for the converters which supply the electrical machine, thus it is sensible to supply each machine module with a separate converter.

Due to easiness and reliability issues, standard two level converters may be a suitable solution, rather than more complex structures, like multilevel or matrix converters. In a two level converter, the DC bus voltage is limited by the switching devices maximum voltage. As regards the current, it can reach high values in high power systems; this is particularly true in Wind Energy Conversion Systems (WECSs) based on PM Synchronous Generators (PMSGs), using full power converters. A high current may limit the switching frequency. Thus, in case of a sinusoidal PWM, it can be useful to exploit not only the linear zone, but also the over modulation (OM) and the square wave (SW) zones, in order to limit the switching frequency.

All the authors are with the Department of Energy at Politecnico di Milano, Via La Masa 34, 20156 Milano, Italy.

antonino.digerlando@polimi.it, khaled.elshawarby@mail.polimi.it, gianmaria.foglia@polimi.it, roberto.perini@polimi.it
Other papers [1], [2] investigate the operation of a multi megawatt and multi modular WECS, based on an Axial Flux Multi Modular (AFMM) PMSG, where each generator module is connected to a two level converter, operating as a PWM rectifier, and the several DC buses are parallelized. Concerning the rectifier (machine-side converters), they are operated with a sinusoidal PWM modulation, and the proposal is applying a suited shift among the carrier signals and the control signals of the several paralleled converter modules, in order to reduce the harmonic content of the DC bus current and of the machine global torque. In these papers, the study concerns separately the operation in SW or in PWM linear zone; in particular, if the converter operates only in SW zone, the square wave output voltages of the several modules are displaced among them, and this requires a mechanical displacement among the machine modules [1]; if the converter operates only in PWM linear zone, the control signals of the several modules (and, thus, the machines modules) are in phase, and only the carrier signals of the several modules are displaced among them [2]. In this paper, the full range operation (linear zone, OM, SW) is proposed.

As for the control of the WECS, usually the control of the machine-side converter (MSC) is in charge of extracting the maximum power from the wind, whereas the control of the grid-side converter (GSC) aims to keep the DC link voltage fixed [3] - [12]; hardly ever the DC-link voltage control is achieved by the MSC instead of the GSC [13]. GSC can also be devoted to some extra functions, such as inertia emulation, energy storage, power quality, grid fault ridethrough capability, and the support to the grid voltage restoration after the fault [14].

In the MSC command strategy, the Maximum Power Point Tracking (MPPT) is combined with several modulation and control techniques: PWM [4], Space Vector Modulation (SVM) [5] sliding mode (SM) nonlinear control [6] [7], rotor field oriented control (RFOC) and direct torque control (DTC) [8] [9]. All the previous solutions adopt a three-leg converter, with 6 bidirectional static switches. However, to reduce the costs, some solutions exist, with a semi-controlled rectifier (only 3 bidirectional static switches) [11] [12].

Among these several solutions, we propose a PWM technique, which exploits also the OM zone. With a fixed DC link voltage $V_{d}$, the OM zone allows to increase the amplitude of the fundamental output voltage: as known, at the limit of the linear zone (i.e., when the modulation index $\mathrm{M}=1$ ), the fundamental amplitude is $\mathrm{V}_{1 \mathrm{a}}=\mathrm{V}_{\mathrm{d}} / 2$, whereas at the end of the over-modulation zone (i.e., in SW operation), $\mathrm{V}_{1 \mathrm{a}}=(4 / \pi) \cdot \mathrm{V}_{\mathrm{d}} / 2$, with an increment of $27 \%$. 
As a drawback, low order harmonics arise: they are mitigated by the proposed suited shifting of the machine modules and of the PWM carrier signals.

In the following, Section II describes the analyzed WECS, Section III illustrates the proposed methodology, Section IV shows the simulation method and results.

\section{SYSTEM DESCRIPTION}

The analysed multi-modular machine is shown in Fig.1.

Several electromagnetically independent AFPM modules are keyed on the same shaft and each of them is connected to a two-level AC-DC converter. The converters are parallel connected at the DC side. The assumed rated data of each module are reported in Table I.

As it can be guessed from the low rated speed in Table I, a direct-drive turbine-generator system is considered. Each module has a reasonably limited rated power, which is compatible with the use of two-level converters.

Moreover, each module has a very low cogging level and torque ripple in sinusoidal operation: thus, in the following the ripple is assumed as due to the converter interface only.

We assume that the electric machine works in Maximum Torque Per Ampere (MTPA) operation (by a phasor representation, internal emf is in phase with phase current).

\section{METHOD DESCRIPTION}

\section{A. Relation Between the Electromechanical Quantities and the Wind Speed}

When a PWM method is adopted to drive the MSC, not always the papers fully and explicitly declare the relation between the wind speed and the electromechanical quantities. As an example [4] proposes the following control strategy:

1) when wind velocity is between cut-in and a transition value $\mathrm{v}_{\mathrm{wt}}$ (stage II), an optimal speed reference is applied in order to obtain maximum power from the wind;

2) when wind velocity is greater than $v_{w t}$, generator speed is held at its rated value by limiting speed reference (stage III); rated torque is obtained at rated wind velocity;

3 ) when wind velocity is higher than rated, power is limited by pitch control. To avoid over rated power excursions due to wind gusts, a constant power reference is obtained by reducing torque (with the increase of rotational speed).

Fig. 2a shows the well-known relations between the electromechanical quantities (machine speed $\Omega$, internal emf $\mathrm{E}$, phase current $\mathrm{I}$, torque $\mathrm{T}$, power $\mathrm{P}$ ) and the wind speed $\left(\mathrm{v}_{\mathrm{w}}\right)$, in traditional operation.

With respect to traditional operation, in our proposal (Fig. 2b), we assume that:

- the rated current and torque do not change;

- the rotating system has a higher rated speed;

- the generator has a higher insulation voltage.

In such a way both the rated wind speed and the rated power can increase.

As shown in Fig. 2b, the traditional rated wind speed $\mathrm{v}_{\text {rated.trad }}$ becomes a transition speed $\mathrm{v}_{\mathrm{wt}}$, and the actual $\mathrm{v}_{\text {rated }}$ becomes higher.

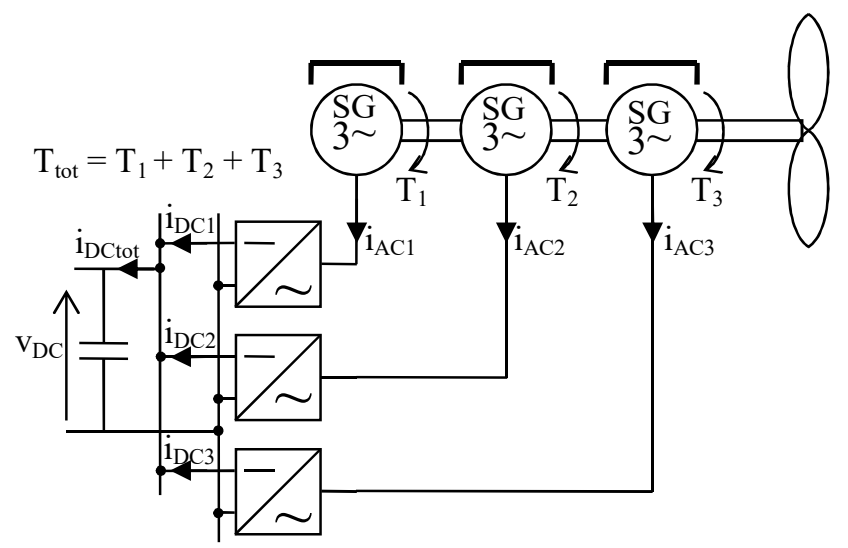

Fig. 1. Scheme of the analyzed system. A multi modular AFPMSG consists of several modules, with each module winding connected to a two-level ACDC converter. The converters are parallel connected at the DC side.

TABLE I

Main Data of Each Module of The AFPM Machine.

\begin{tabular}{c|c}
\hline Rated $P_{n}[\mathrm{~kW}], I_{n}[\mathrm{~A}], N_{n}[\mathrm{rpm}]$ & $1000 ; 657 ; 17$ \\
\hline $\begin{array}{c}\text { Line to neutral EMF at rated speed } E_{0}\left[\mathrm{~V}_{R M S}\right] \\
\text { (sinusoidal waveform) }\end{array}$ & 507.5 \\
\hline No of poles $p$, rated frequency $f_{n}[\mathrm{~Hz}]$ & $104 ; 14.73$ \\
\hline Stator flux linkage due to PM, $\Psi_{P M}=E_{0} /\left(2 \pi f_{n}\right)[\mathrm{Wb}]$ & 5.48 \\
\hline Phase resistance $R[\mathrm{~m} \Omega]$ & 16.15 \\
\hline Synchronous inductance $(\mathrm{d}, \mathrm{q}$ axes) $L[\mathrm{mH}]$ & 3.132 \\
\hline Axial length (one module) $[\mathrm{m}]$ & 0.336 \\
\hline External diameter $[\mathrm{m}]$ & 5.000 \\
\hline Rotor inertia $\left[\mathrm{kg} \mathrm{m}{ }^{2}\right]$ & $3.707 \cdot 10^{4}$ \\
\hline
\end{tabular}

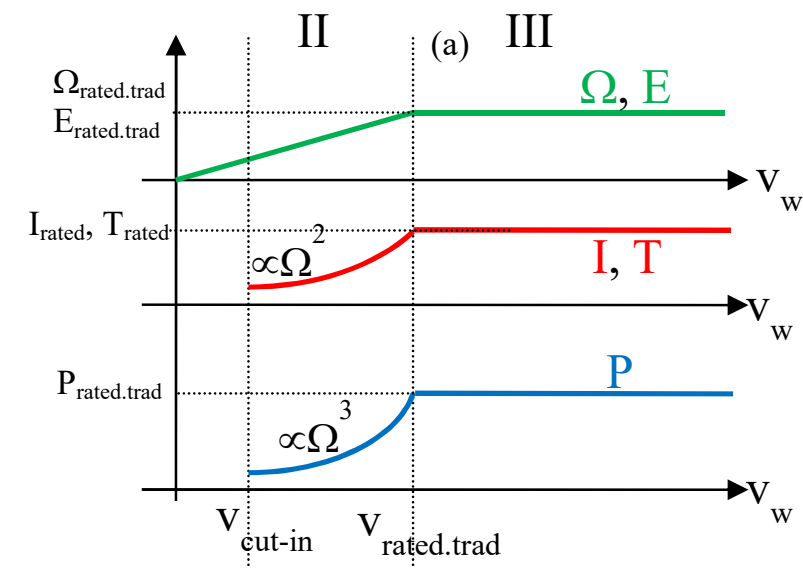

(b)

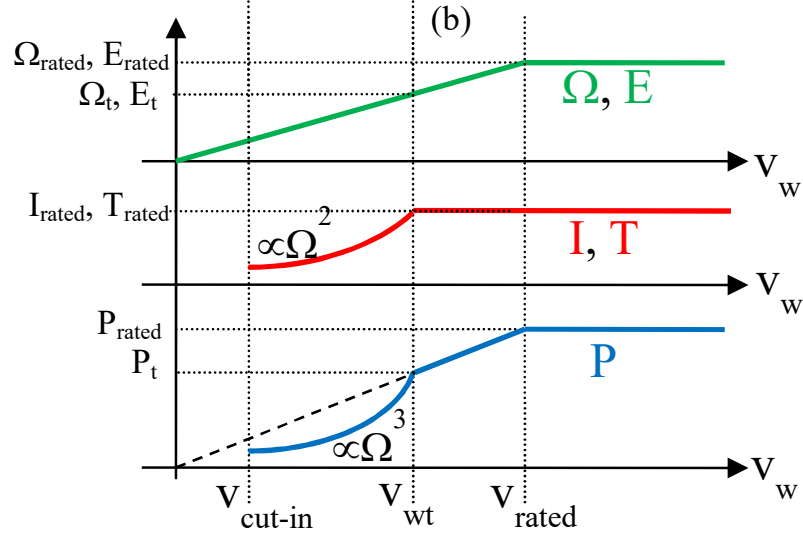

Fig. 2. Relations between the electromechanical quantities (machine speed $\Omega$, internal emf E, phase current I, torque T, power P) and the wind speed $\left(\mathrm{V}_{\mathrm{w}}\right)$, in traditional operation (a) and in our proposal (b). 
From cut-in speed $\mathrm{v}_{\text {cut-in }}$ up to $\mathrm{v}_{\mathrm{wt}}$, nothing changes with respect to traditional operation. For $\mathrm{v}_{\mathrm{w}}>\mathrm{v}_{\mathrm{wt}}$, up to the actual $\mathrm{V}_{\text {rated }}$, current and torque are kept constant at their rated values $I_{\text {rated }}, T_{\text {rated }}$, all the other quantities are proportional to $\mathrm{v}_{\mathrm{w}}$; clearly, a pitch regulation is required. At $\mathrm{v}_{\text {rated }}$, also $\Omega$, $\mathrm{E}, \mathrm{P}$ reach their rated value (higher than in the traditional operation), so all the quantities are at their rated value. For higher wind speeds $v_{w}>v_{\text {rated }}$, the pitch regulation allows to keep all the quantities constant.

Therefore, assumed a machine with higher voltage and higher speed ratings, the proposed operation allows, with a fixed turbine, to increase the power. On the other hand, by means of SW operation of the inverter, the higher voltage rating does not need an increase of the DC link voltage, thus the same electronic switches can be adopted as if the converter operated in the PWM linear zone.

\section{B. Relation Between the Modulation Function and the Amplitude Modulation Index}

Let us consider a naturally-sampled PWM. Let $\mathrm{f}_{\text {con }}, \mathrm{f}_{\text {tri }}$, $\mathrm{V}_{\text {con.p }}, \mathrm{V}_{\text {tri.p }}$, be the frequency and the peak value of the control signal and triangular carrier signal, $\mathrm{V}_{\mathrm{d}}$ be the DC bus voltage, $\mathrm{V}_{\text {out1.p }}$ be the fundamental output voltage peak value. The amplitude modulation index $\mathrm{M}$, the modulation function $\mathrm{MF}$, the frequency modulation index $\mathrm{m}_{\mathrm{f}}$ are defined as

$$
\mathrm{M}=\mathrm{V}_{\text {con.p }} / \mathrm{V}_{\text {tri.p }}, \mathrm{MF}=\mathrm{V}_{\text {out } 1 . \mathrm{p}} /\left(0.5 \mathrm{~V}_{\mathrm{d}}\right), \mathrm{m}_{\mathrm{f}}=\mathrm{f}_{\text {tri }} / \mathrm{f}_{\text {con }} .
$$

(in three phase systems, usually $\mathrm{m}_{\mathrm{f}}$ is odd and multiple of 3 ). As known [14], in the linear PWM zone $(\mathrm{M}<1), \mathrm{MF}=\mathrm{M}$ occurs, and in the SW operation $\left(M>M_{L I M}\right), M F=4 / \pi$. The limit value $\mathrm{M}_{\mathrm{LIM}}$ which starts the $\mathrm{SW}$ operation depends on $\mathrm{m}_{\mathrm{f}}$, according to the relation $\mathrm{M}_{\mathrm{LIM}}\left(\mathrm{m}_{\mathrm{f}}\right)=1 / \sin \left(\frac{3}{2} \frac{\pi}{m_{f}}\right)$.

In the OM zone $\left(1<\mathrm{M}<\mathrm{M}_{\text {LIM }}\right)$, MF tends asymptotically to $4 / \pi$, according to the expression [14]

$$
M F_{\text {OMasymp }}(M)=\frac{2}{\pi}\left[M \cdot \operatorname{asin}\left(\frac{1}{M}\right)+\sqrt{1-\left(\frac{1}{M}\right)^{2}}\right] .
$$

Actually, this is only a theoretical expression: as cited, $\mathrm{MF}=$ $4 / \pi$ for $M>M_{\text {LIM }}$. In order to avoid discontinuities in $\mathrm{MF}(\mathrm{M})$ values, we suggest that $\mathrm{MF}(\mathrm{M})$ follows (2):

$$
M F(M)=\left\{\begin{array}{cc}
M F_{\text {OMasymp }}(M) & 1<M<M_{\text {bound }} \\
\text { linear interpolation } & M_{\text {bound }}<M<M_{L I M} \\
4 / \pi & M>M_{L I M}
\end{array}\right.
$$

$\mathrm{M}_{\text {bound }}$ is a suited value, for instance $\mathrm{M}_{\text {bound }}=0.7 \cdot \mathrm{M}_{\mathrm{LIM}}$; the value of $\mathrm{MF}(\mathrm{M})$ at $\mathrm{M}=\mathrm{M}_{\text {bound }}$ is defined $\mathrm{MF}_{\text {bound }}$ and equals

$$
\mathrm{MF}_{\text {bound }}=\mathrm{MF}_{\text {OMasympt }}\left(\mathrm{M}_{\text {bound }}\right) \text {. }
$$

The $M F(M)$ linear interpolation ranges from $M_{\text {bound }}$ to $4 / \pi$. Fig. 3 shows the behavior of $\mathrm{MF}(\mathrm{M})$ as expressed by (1) and (2), for $\mathrm{m}_{\mathrm{f}}=15$ and $\mathrm{M}_{\text {bound }}=0.7 \cdot \mathrm{M}_{\mathrm{LIM}}$.

\section{Relation Between the Amplitude Modulation Index and the Wind Speed}

Let us define the per unit wind speed $v=v_{w} / v_{\text {rated }}$ (equal to the per unit rotation speed, for $\mathrm{v}_{\mathrm{w}}<\mathrm{v}_{\text {rated }}$ ).

In order to link Fig. $2 b$ and Fig. 3, we assume $M=1$ at $v_{w}=$ $\mathrm{v}_{\mathrm{wt}}\left(v=\mathrm{v}_{\mathrm{wt}} / \mathrm{v}_{\text {rated }}=v_{\mathrm{t}}\right)$, and $\mathrm{M}=\mathrm{M}_{\text {LIM }}$ at $\mathrm{v}_{\text {rated }}(v=1)$. To comply with the voltage linear behaviour in Fig. $2 b, v_{t}$ must equal $\pi / 4$ (at the OM zone boundaries, the ratio between the speeds equals the ratio between the voltages, i.e. $\pi / 4$ ).

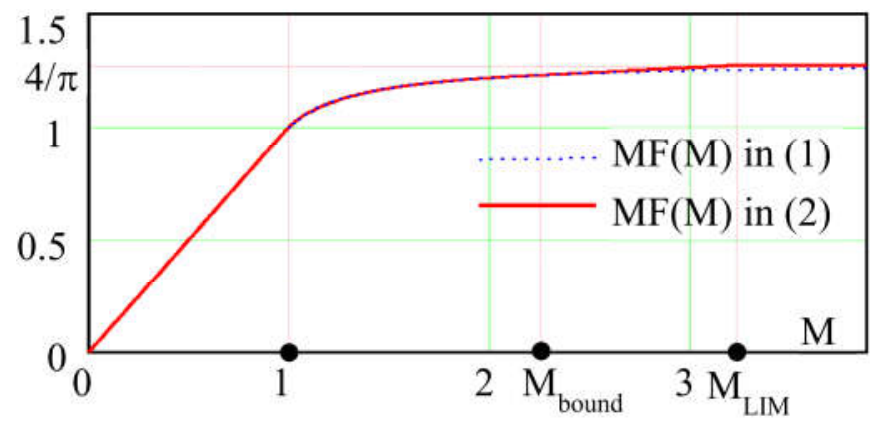

Fig. 3. $\mathrm{MF}(\mathrm{M})$ as given by (1) and (2), for $\mathrm{m}_{\mathrm{f}}=15$ and $\mathrm{M}_{\text {bound }}=0.7 \cdot \mathrm{M}_{\mathrm{LIM}}$.

According to Fig. 2b, the behaviour of $\mathrm{E}$ and $\mathrm{I}_{1}$ (rms value of the fundamental phasor) as a function of $v$ is expressed by

$$
\mathrm{E}(v)=v \cdot \mathrm{E}_{\text {rated }} ; \quad I_{1}(v)=\left\{\begin{array}{cc}
I_{\text {rated }} \cdot\left(\frac{v}{v_{t}}\right)^{2} & v<v_{t} \\
I_{\text {rated }} & v>v_{t}
\end{array}\right.
$$

According to the MTPA ( $\mathrm{E}$ is in phase with $\mathrm{I}_{1}$ ), the expression of the machine voltage $\mathrm{V}_{1}$ ( $\mathrm{rms}$ value of the fundamental phasor) as a function of $v$ is

$$
V_{1}(v)=\sqrt{\left(E(v)-R \cdot I_{1}(v)\right)^{2}+\left(v \cdot 2 \pi f_{n} \cdot L \cdot I_{1}(v)\right)^{2}}
$$

It can be verified that, as for the amplitude, the voltage is very close to the emf, that is $V_{1}(v) \approx E(v)$.

Fig. 3 gives the relation $\operatorname{MF}(\mathrm{M})$, that is $\mathrm{V}_{1}(\mathrm{M})$; by reversing it, relation $\mathrm{M}\left(\mathrm{V}_{1}\right)$ can be obtained; the latter, together with (4), yields the relation $\mathrm{M}(v)$. This relation gives, for any wind speed, the required modulation index.

\section{Shifting Method}

To illustrate the fundamentals of the method, let us consider the basic structure of a three-phase active rectifier (Fig.4): each generator phase is modelled as a series $R-L-e$ circuit, point $z$ represents the virtual midpoint of the capacitor on the DC side, points $A, B, C$ are the mid points of the converter legs, point $o$ is the star point of the generator.

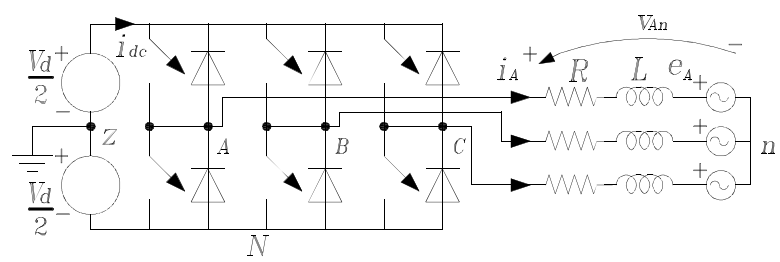

Fig. 4. Equivalent circuit for one module of the AFPM machine and its converter. The load sign convention is used.

Equation (5) reports the analytical harmonic solution for the double-edge naturally-sampled PWM of the three phase converter with sinusoidal modulation [14], in PWM linear zone. It expresses the pole voltage $v_{K z}, K=A, B, C$, i.e. voltage across points $A, B, C$ and the capacitor midpoint $z$.

$$
\begin{gathered}
v_{K z}=\left(V_{d} / 2\right) \cdot M \cos \left(\omega_{1} t-\theta\right)+ \\
+\frac{2 V_{d}}{\pi} \sum_{m=1}^{\infty} \sum_{n=-\infty}^{\infty} \frac{1}{m} J_{j}\left(m \frac{\pi}{2} M\right) \cdot \sin \left([m+n] \frac{\pi}{2}\right) . \\
\cdot \cos \left(m\left[\omega_{c} t+\psi_{c}\right]+n\left[\omega_{1} t-\theta+\psi_{m}\right]\right)
\end{gathered}
$$


In (5) $J_{j}$ is the Bessel function of the first kind and order $j$; $m$ is the carrier index and $n$ is the base band index for the $h$ th harmonic component, due to the PWM modulation: $h=$ $m \cdot m_{f}+n ; \omega_{1}$ is the fundamental angular frequency; $\omega_{c}$ is the carrier angular frequency: $\omega_{c}=m_{f} \cdot \omega_{1}$. The angle $\psi_{c}$ is the phase of the carrier signal applied to the converter, with reference to the instant $t=0$ (this time instant is the same for the whole system); $\psi_{c}$ is expressed in electrical degrees of the carrier (Fig.5a). The angle $\psi_{m}$ is the phase of the sinusoidal control signal applied to the converter, measured with respect to $t=0 ; \psi_{m}$ is expressed in electrical degrees of the control signal (Fig.5b). $\theta$ is the displacement among the phases: $\theta=0$ for $v_{A z}, \theta=-2 \pi / 3$ for $v_{B z}, \theta=-4 \pi / 3$ for $v_{C z}$.
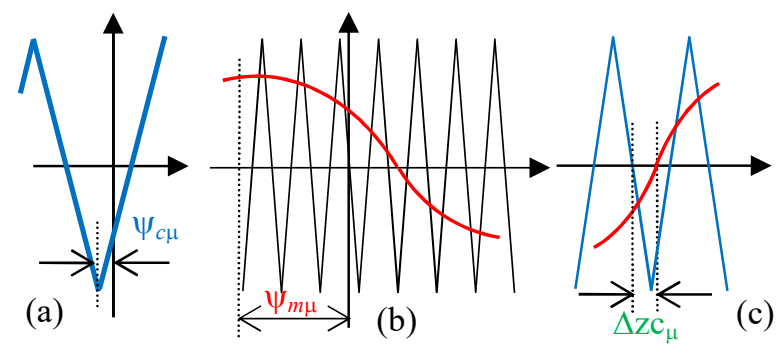

Fig. 5. (a): definition of angle $\psi_{c \mu}$ : phase of the carrier signal applied to the converter $\mu$, with reference to the instant $\mathrm{t}=0$ (time instant $\mathrm{t}=0$ is the same for the whole system); the carrier signal reference point is its minimum value; $\psi_{c \mu}$ is expressed in electrical degrees of the carrier. (b): definition of angle $\psi_{m \mu}$ : phase of the control signal applied to the converter $\mu$, with reference to $\mathrm{t}=0$; the control signal is assumed as a cosine function; $\psi_{m \mu}$ is expressed in electrical degrees of the control signal. (c): definition of the displacement $\Delta \mathrm{zc}_{\mu}$ between the zero crossing of the control signal and the zero crossing of the carrier signal (with opposite slopes), in converter $\mu$.

Assume that the multi module WECS has $N_{\mu}$ modules; then, Fig. 4 can be regarded as the generic $\mu$-th module $(\mu=1,2 \ldots$ $N_{\mu}$ ). Due to (5), in each module and for each harmonic, the phase of the generic harmonic current, and thus the phase of the generic harmonic torque, depend on a linear combination of the displacement angles $\psi_{c \mu}$ and $\psi_{m \mu}$ of that module: the resultant displacement angle $\psi_{\mu}$ can be expressed as

$$
\psi_{\mu}=\mathrm{k}_{\mathrm{m}} \cdot \psi_{m \mu}+\mathrm{k}_{\mathrm{c}} \cdot \psi_{c \mu},
$$

where $\mathrm{k}_{\mathrm{m}}$ an $\mathrm{k}_{\mathrm{c}}$ are analytically calculated coefficients [15] (see an example of their values in Table II); note that $\mathrm{k}_{\mathrm{m}}$ and $\mathrm{k}_{\mathrm{c}}$ differ from $m$ and $n$ in (5), since (5) refers to harmonic voltages, (6) to harmonic currents and torques). In the global WECS, the output dc currents and the torques of the single modules add up. Thus by properly choosing the control angles $\psi_{c \mu}$ and $\psi_{m \mu}$, selected harmonics can be eliminated from the global waveform. In particular, as a general rule, in order to cancel a definite harmonic, the related displacement $\psi_{\mu}$ is to be set as follows:

for 2 modules $\left(N_{\mu}=2\right): \psi_{2}-\psi_{1}=180^{\circ}$;

for 3 modules $\left(N_{\mu}=3\right): \psi_{2}-\psi_{1}=\psi_{3}-\psi_{2}=120^{\circ}$.

As far as the PWM linear zone is considered (i.e. $M<1$ ), the control signals of the several modules could be in phase (i.e., $\psi_{m \mu}=0$ for all modules), since the harmonic elimination can be carried out just by setting the $\psi_{c \mu}$ angles of the several modules; such analysis is developed in [2]. But, when the
SW operation is taken into account, angle $\psi_{c \mu}$ no more make sense, thus angles $\psi_{m \mu}$ must be used. Three matters arise.

The first is as follows: each module $\mu$ has a distinctive value of angle $\psi_{m \mu}$, different from the other modules; this requires either of the two following solutions:

- the stator flux space vectors of the several modules are in phase, and the rotor flux space vectors of the several modules are not in phase; this is obtained by aligning the stator modules and by mechanically shifting the rotors during the machine assembly process, as assumed in [1] (see Fig. 2 in [1] and Fig.6a);

- the opposite: the rotor flux space vectors of the several modules are in phase, and the stator flux space vectors of the several modules are not in phase; this is obtained by aligning the rotor modules and by mechanically shifting the module stator windings axes during the assembly process (by adopting suited mechanical shifting angles of the windings, multiples of the angle between adjacent teeth); this is the condition assumed in this paper (see Fig.6b).

Notice that, in order to obtain an angle shift among the module stator fluxes, it is not possible to change the torque angle: in fact, the latter is set by adopting the desired control method, namely MTPA operation; therefore in each module the stator flux is kept in quadrature with respect to the rotor flux of that module.

The second matter is: if the machine operates in a wide speed range, so that both PWM and SW controls are adopted for the converters, the stator windings (or the rotors) displacements are set during the assembly process. This means that the angles $\psi_{m \mu}$ are different from 0 , and such condition must be taken into account in the linear zone operation. This situation is different from [2], where, as previously cited, for all modules $\psi_{m \mu}=0$ occurs.

The third matter regards the definition of the angle $\psi_{m \mu}$ values (since they are required for the machine assembly). The same criterion adopted in [1] will be used here: $\psi_{m \mu}$ angles are chosen in order to cancel the lowest order torque harmonic, occurring in the SW operation. Once set $\psi_{m \mu}$ values, $\psi_{c \mu}$ values will be set, according to different criteria, which are discussed in the following.

\section{E. Definition of $\psi_{\mathrm{m} \mu}$ Angles}

As previously explained, the $\psi_{m \mu}$ angles are chosen with reference to SW operation (clearly, in SW operation, $\psi_{m \mu}$ becomes the phase of the square wave, too). By adopting the same procedure carried out in [1], the expressions of the harmonic torques can be developed. The same conclusion of [1] holds: in SW operation, the lowest harmonic is the $6^{\text {th }}$, and in order to cancel it, the following choices are suitable:

for 2 modules $\left(N_{\mu}=2\right): \psi_{m 1}=0^{\circ}, \psi_{m 2}=30^{\circ}$

for 3 modules $\left(N_{\mu}=3\right): \psi_{m 1}=0^{\circ}, \psi_{m 2}=20^{\circ}, \psi_{m 3}=40^{\circ}$

In [1], $\psi_{m \mu}$ was the space displacement of the $\mu$-th rotor, referred to the stator reference axis (same reference for all the stators) (see Fig.6a); here, $\psi_{m \mu}$ is the space displacement 
of the stator reference axis of the $\mu$-th stator with reference to the rotor axis (same for all the rotors) (see Fig.6b); both in [1] and here, $\psi_{m \mu}$ is also the time electrical displacement of the square wave in the converter of the $\mu$-th module, with reference to the instant $t=0$ (the same for the whole system).

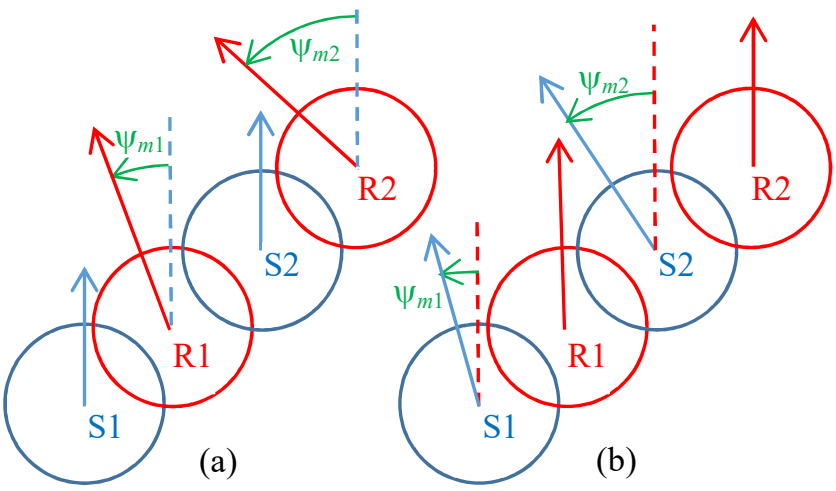

Fig.6. Schematic drawing of two module systems, with stator and rotor magnetic axes: (a) aligned stator axes and displaced rotor axes; (b) aligned rotor axes and displaced stator axes.

\section{F. Definition of $\psi_{\mathrm{c \mu}}$ Angles - Criterion 1: Elimination of One Selected Harmonic.}

The $\psi_{c \mu}$ angles are chosen with reference to PWM linear zone. By adopting the same procedure carried out in [2], the expressions of the harmonic torques can be developed. The same conditions assumed in [2] are here considered: the Bessel function $J_{j}$ is simplified to the first 3 terms of the Taylor series (this is a suited approximation for $M \leq 1$ ), and only some harmonics limited to $m=1,2$ and $n=0, \pm 1, \pm 2$, \pm 3 , are considered. Since in [2] all the modules are in phase $\left(\psi_{m \mu}=0 \forall \mu\right)$, the results are here different. The expression of the DC bus current ripple due to the converter of the $\mu$-th module is reported in Appendix A-1 of [15]. It is summarized in Table II, which reports, for each group of harmonics $h$, their amplitude, the coefficient $\mathrm{k}_{\mathrm{c}}$ in (6) (which gives the share of displacement due to the shift in the carrier signal), the coefficient $\mathrm{k}_{\mathrm{m}}$ in (6) (which gives the share of displacement due to the shift in the control signal, obtained by means of the stator windings mechanical shifting). Table II reads as follows: with the considered limitations ( $m=1,2$ and $n=0, \pm 1, \pm 2, \pm 3$ ), the DC current harmonic $h=m_{f}-3$, produced by the $\mu$-th converter, has 4 terms (due to the interaction of several $\mathrm{AC}$ voltage and current harmonics, for example: the term whose amplitude is $V_{1} I_{m f-2}$, is due to the interaction between the $1^{\text {st }}$ harmonic of the voltage and the harmonic $h=m_{f}-2$ of the current); all these 4 terms have the same displacement, equal to $\psi_{\mu}=\psi_{c \mu}-3 \psi_{m \mu}$.

Let us assume to cancel the lowest order harmonic $h=m_{f}$ - 3; then, according to the general rule (7), and to the previous choice of $\psi_{m \mu}(8)$, the related choice of $\psi_{c \mu}$ are:

for 2 modules $\left(N_{\mu}=2\right): \psi_{c 1}=\psi_{1}+3 \psi_{m 1}=0^{\circ}$; $\psi_{c 2}=\psi_{2}+3 \psi_{m 2}=180^{\circ}+3 \cdot 30^{\circ}=270^{\circ}$;

for 3 modules $\left(N_{\mu}=3\right): \psi_{c 1}=\psi_{1}+3 \psi_{m 1}=0^{\circ}$;

$\psi_{c 2}=\psi_{2}+3 \psi_{m 2}=120^{\circ}+3 \cdot 20^{\circ}=180^{\circ}$;

$\psi_{c 3}=\psi_{3}+3 \psi_{m 3}=240^{\circ}+3 \cdot 40^{\circ}=0^{\circ}$
Note that Table II shows that each harmonic has several terms: for example, harmonics $h=m_{f}-3$ and $h=m_{f}+3$ both have 4 terms, whereas harmonic $h=2 m_{f}$ has 6 terms; moreover, the several terms have different amplitudes and phases. Thus, there's no guarantee that the lowest harmonic has the highest amplitude. Therefore, before applying this criterion, a survey must be done, to check which harmonic has the highest amplitude.

TABLE II

SUMMARY OF THE DC BUS CURRENT RIPPLE EXPRESSION

\begin{tabular}{c|l|c|c}
\hline \multicolumn{1}{c|}{$h$} & \multicolumn{1}{|c|}{$I_{d c h} \cdot \frac{V_{d}}{3}$} & $\begin{array}{c}\mathrm{k}_{c} \\
\text { in (6) }\end{array}$ & $\begin{array}{c}\mathrm{k}_{m} \\
\text { in (6) }\end{array}$ \\
\hline$m_{f}-3$ & $\begin{array}{l}V_{1} I_{m f-2}, V_{m f-2} I_{1} \\
V_{m f+2} I_{2 m f-1}, V_{2 m f-1} I_{m f+2}\end{array}$ & 1 & -3 \\
\hline$m_{f}+3$ & $\begin{array}{l}V_{1} I_{m f+2}, V_{m f+2} I_{1} \\
V_{m f-2} I_{2 m f+1}, V_{2 m f+1} I_{m f-2}\end{array}$ & 1 & 3 \\
\hline $2 m_{f}$ & $\begin{array}{l}V_{1} I_{2 m f-1}, V_{2 m f-1} I_{1} \\
V_{1} I_{2 m f+1}, V_{2 m f+1} I_{1} \\
V_{m f-2} I_{m f+2}, V_{m f+2} I_{m f-2}\end{array}$ & 2 & - \\
\hline $3 m_{f}-3$ & $V_{m f-2} I_{2 m f-1}, V_{2 m f-1} I_{m f-2}$ & 3 & -3 \\
\hline $3 m_{f}+3$ & $V_{m f+2} I_{2 m f+1}, V_{2 m f+1} I_{m f+2}$ & 3 & 3 \\
\hline $4 m_{f}$ & $V_{2 m f-1} I_{2 m f+1}, V_{2 m f+1} I_{2 m f-1}$ & 4 & - \\
\hline
\end{tabular}

NOTE: $V_{h}, I_{h}$ are the RMS values of the $h$-th voltage and current harmonic. Their expressions can be found in Appendix A-1 of [15].

\section{G. Definition of $\psi_{c \mu}$ Angles - Criterion 2: Lowering Global THD.}

If the system operates for long time in a definite condition, and a definite harmonic is dangerous, the previous criterion is the best suited. In a WECS, usually the operating conditions continuously change, so eliminating a single harmonic may not be the best choice. A better choice could be the $\psi_{c \mu}$ value which minimizes the global THD. Since a direct relation between the $\psi_{c \mu}$ value and the global THD does not exist, the solution should be found by a survey of different $\psi_{c \mu}$ values.

\section{H. Definition of $\psi_{c \mu}$ Angles - Criterion 3:}

Same PWM Waveforms in the Several Modules.

By applying the two previous criteria, in general the value of the displacement $\Delta \mathrm{zc}_{\mu}$ between the zero crossing of the carrier and the zero crossing of the control signal in module $\mu$ (Fig.5c) is different from the corresponding value in the other modules; this implies that the intersections between the carrier signal and the control signal occur in different points in each module, thus the PWM waveforms are different in the several modules. As a consequence, the modules behave differently as regards the harmonic contents; this introduces a dissymmetry in the system, and may increase the global harmonic content in the whole operating range. Thus, in a system like a WECS, where a steady state operation does not exist, a suited constraint seems to be the symmetry among the several modules: this implies that $\Delta \mathrm{zc}_{\mu}$ is the same in all the modules. This constraint (together with (8) ) directly sets the value of the $\psi_{c \mu}$ angles: they must equal $\psi_{c \mu}=\Delta \mathrm{zc}_{\mu}+\mathrm{m}_{\mathrm{f}}$. $\cdot \psi_{m \mu}$ (expressed in electrical angles of the carrier signal). As 
an example, in the following, the case $N_{\mu}=3, \mathrm{~m}_{\mathrm{f}}=15, \Delta \mathrm{zc}_{\mu}$ $=0$ is considered, which implies $\psi_{c 1}=0, \psi_{c 2}=300^{\circ}, \psi_{c 3}=$ $600^{\circ}=240^{\circ}$.

\section{Simulation Results}

Up to now, just the steady state operation has been studied, by means of the state variable model of the PMSM. The inverter PWM voltage waveforms are obtained by the traditional sinusoidal PWM: intersection between the carrier signal (triangular waveform, amplitude $=1$ ) and the control signal ( sinusoidal waveform, amplitude $=M(v)$ ). For several wind speed values (i.e., several values of the variable $v$ ), the PWM voltage waveforms have been found, the state variable equations have been solved, and the waveforms of the main electromechanical quantities (Fig. 1) have been evaluated:

- for each module: AC current $i_{A C \mu}, D C$ current $i_{D C \mu}, D C$ current ripple $i_{r \mu}$, torque $T_{\mu}$, torque ripple $T_{r \mu}$;

- for the global system: DC current $\mathrm{i}_{\mathrm{DCtot}}$, DC current ripple $\mathrm{i}_{\text {rtot }}$, torque $\mathrm{T}_{\text {tot }}$, torque ripple $\mathrm{T}_{\text {rtot }}$.

On the basis of the waveforms, some global quantities have been calculated:

- for each module: the peak-to-peak ripple $\Delta \mathrm{T}_{\mu}$ and the average value $\mathrm{T}_{\mathrm{av} \mu}$ of the electromagnetic torque, and their ratio $\Delta \mathrm{T}_{\mu} / \mathrm{T}_{\mathrm{av} \mu}$; the RMS value $\mathrm{I}_{\mathrm{r} \mu}$ of the $\mathrm{DC}$ current ripple, the average value $\mathrm{I}_{\mathrm{av} \mu}$ of the DC current, and their ratio $\mathrm{I}_{\mathrm{r} \mu} / \mathrm{I}_{\mathrm{av} \mu}$;

- for the global system: the peak-to-peak ripple $\Delta \mathrm{T}_{\text {tot }}$ and the average value $T_{\text {avtot }}$ of the electromagnetic torque, and their ratio $\Delta \mathrm{T}_{\text {tot }} / \mathrm{T}_{\text {avtot }}$; the RMS value $\mathrm{I}_{\text {rtot }}$ of the $\mathrm{DC}$ current ripple, the average value $\mathrm{I}_{\text {avtot }}$ of the $\mathrm{DC}$ current, and their ratio $\mathrm{I}_{\mathrm{rtot}} / \mathrm{I}_{\text {avtot }}$.

All the evaluations can be repeated for several values of $\psi_{c \mu}$ angles, according to the criteria previously discussed, in order to verify the best choice. Note that $\psi_{m \mu}$ angles are fixed, according to the optimum value defined in (8). In the following some results are reported, for the case of 3 modules $\left(N_{\mu}=3\right)$, with $\mathrm{m}_{\mathrm{f}}=15$. As an example, the $3^{\text {rd }}$ criterion is adopted (same PWM waveforms), with $\Delta \mathrm{zc}_{\mu}=0$. As previously explained, $\psi_{c 1}=0, \psi_{c 2}=300^{\circ}, \psi_{c 3}=240^{\circ}$

Fig.s 7 and 8 show the waveforms of the torque and the DC currents, both for the single modules and for the resultant system (see Fig. 1 for the definition of the quantities); the resultant torque and current are divided by 3 . Only $1 / 6$ of the period is shown. It is apparent that the shifting method highly reduces the ripple: if the shifting were not applied, the quantities of the three modules would be in phase, and the resultant quantity (divided by 3) would have the same amplitude as the single module quantity. The reduction occurs both in PWM linear zone (Fig. 7) and in SW (Fig. 8).

Fig.s 9 and 10 show the behavior, in the whole speed range, of the main performance indexes previously defined, namely the torque ripple and the DC current ripple, for one of the modules (the $2^{\text {nd }}$ ) and for the global system. The ratios $\mathrm{I}_{\mathrm{r} 2} / \mathrm{I}_{\mathrm{av} 2}$ and $\mathrm{I}_{\mathrm{rtot}} / \mathrm{I}_{\mathrm{avtot}}$ are the THDs of the DC current in the $2^{\text {nd }}$ module and in the global DC bus. Again the improvement due to the shifting method is apparent.

Fig.s 11 and 12 show, in all the speed range, the p.u. value of the RMS current and torque harmonics of the $2^{\text {nd }}$ module, for the main harmonics in Table II. In the considered case, for the global system, all these harmonics disappear, except the harmonic with order $\mathrm{m}_{\mathrm{f}}+3$, which survives, and has the same p.u. value as in the $2^{\text {nd }}$ module $\left(\mathrm{T}_{\text {htot }} / \mathrm{T}_{\text {avtot }}=\mathrm{T}_{\mathrm{h} 2} / \mathrm{T}_{\text {av2 }}\right.$ for $\mathrm{h}=\mathrm{m}_{\mathrm{f}}+3 ; \mathrm{T}_{\mathrm{htot}} \approx 0$ for other $\mathrm{h}$ values).
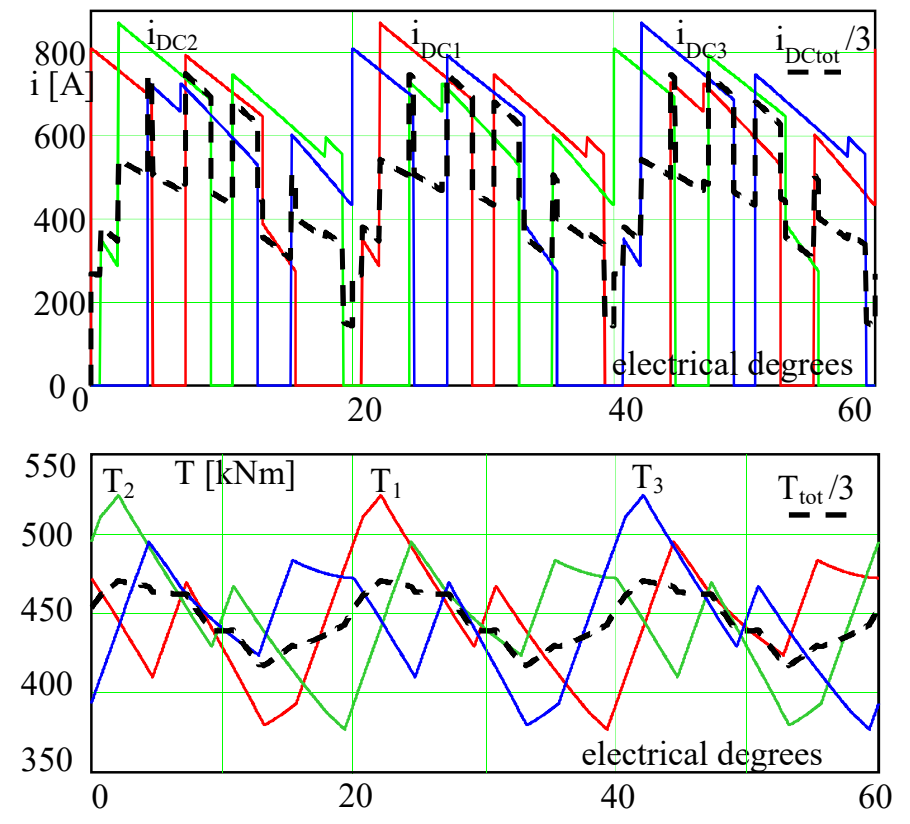

Fig. 7. Current and torque waveforms, for the single modules $\left(i_{D C 1} i_{D C 2} i_{D C 3}\right.$ $\mathrm{T}_{1} \mathrm{~T}_{2} \mathrm{~T}_{3}$ ), and for the resultant system ( $\mathrm{i}_{\mathrm{DCtot}} / 3, \mathrm{~T}_{\text {tot }} / 3$ ), in linear PWM zone.
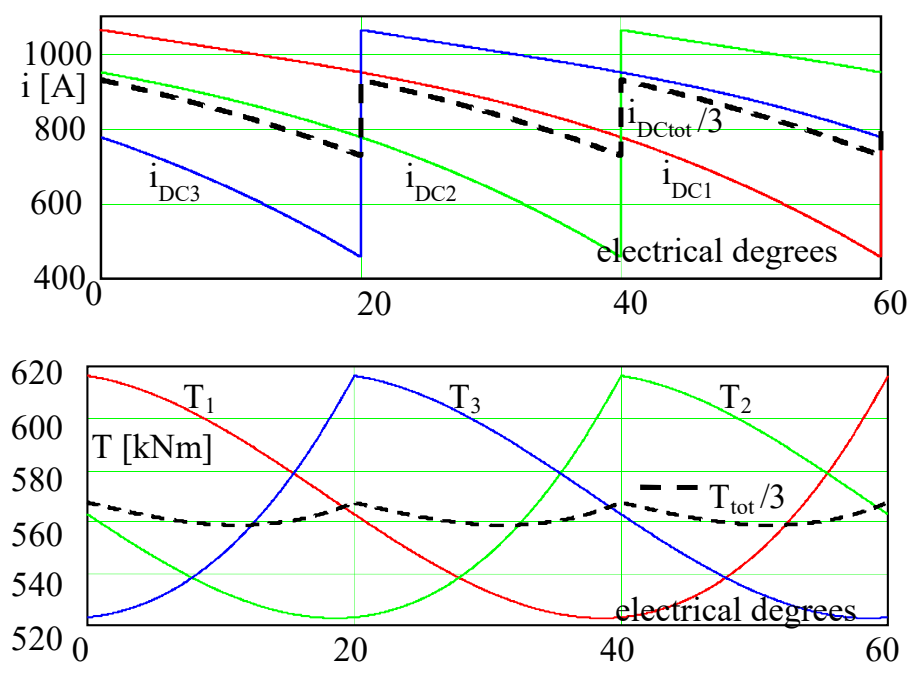

Fig. 8. Current and torque waveforms, for the separate modules ( $i_{D C 1} i_{D C 2}$ $\left.\mathrm{i}_{\mathrm{DC} 3} \mathrm{~T}_{1} \mathrm{~T}_{2} \mathrm{~T}_{3}\right)$, and for the resultant system $\left(\mathrm{i}_{\mathrm{DCtot}} / 3 \mathrm{~T}_{\text {tot }} / 3\right)$, in $\mathrm{SW}$ operation.

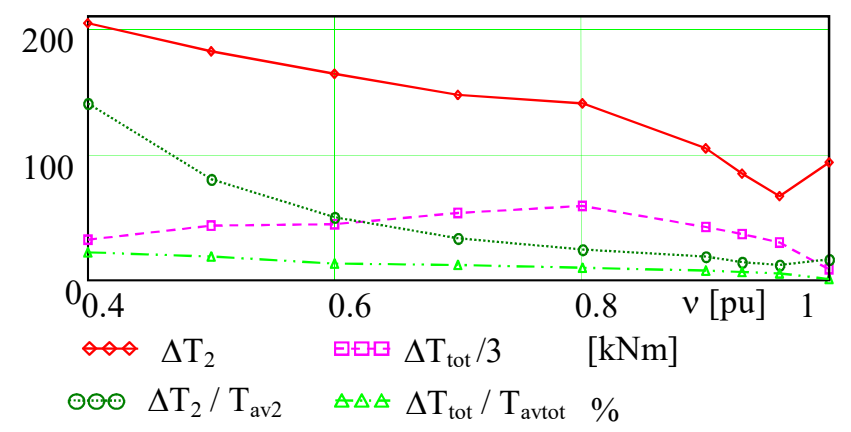

Fig. 9. Peak to peak torque ripple, for the $2^{\text {nd }}$ module, and for the resultant system, both in magnitude and in percentage, in the whole speed range. 


\section{CONCLUSION}

A method has been proposed, aimed to reduce both the torque ripple and the dc current ripple in the machine side converter, in a multi megawatt WECS, based on a PMSG which has a multi modular structure. The method uses not only the PWM linear zone, but exploits also the over modulation and square wave zone. Many simulations of the steady state operation have been carried out, and all of them confirm that the method succeeds in lowering the ripples.

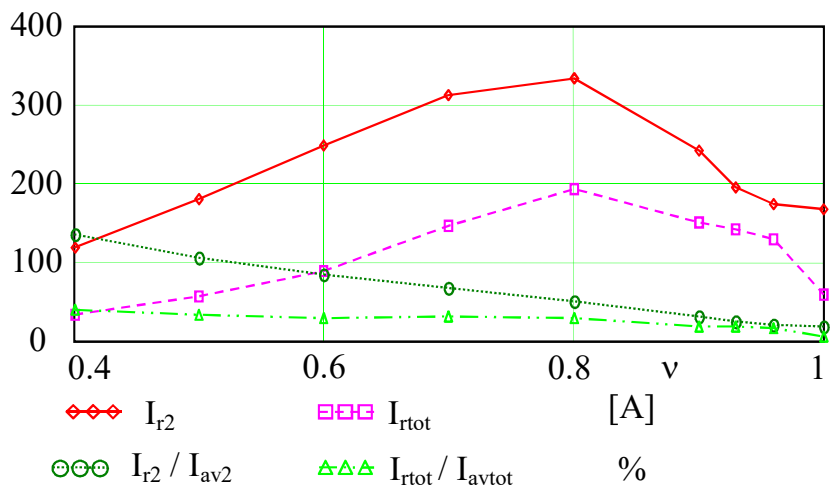

Fig. 10. RMS value of the DC current ripple, for the $2^{\text {nd }}$ module, and for the resultant system, both in magnitude and in [\%], in the whole speed range.

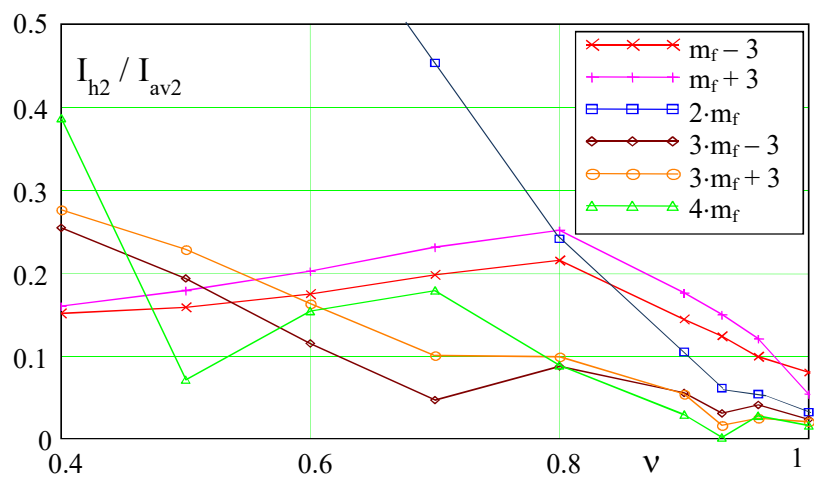

Fig. 11. p.u. RMS value of the DC current harmonics in the $2^{\text {nd }}$ module (ratio between the harmonic RMS value $\mathrm{I}_{\mathrm{h} 2}$ and the average value $\mathrm{I}_{\text {av2 }}$ ), in the whole speed range. The main harmonics of Table II are considered.

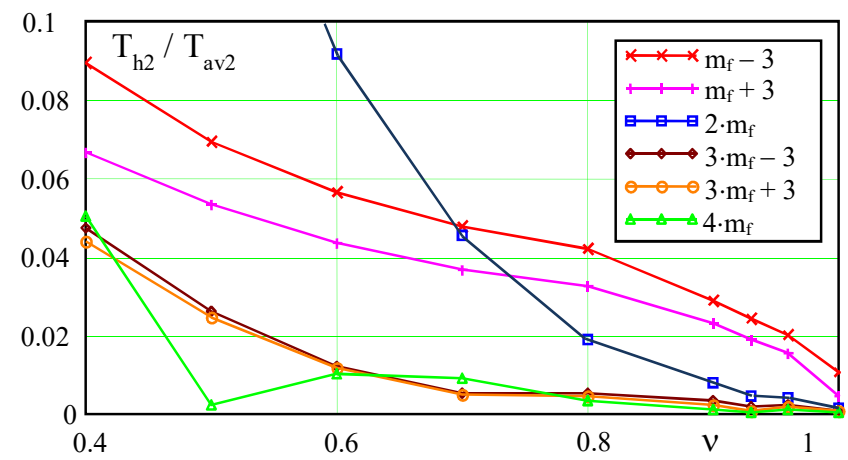

Fig. 12. p.u. RMS value of the torque harmonics in the $2^{\text {nd }}$ module (ratio between the generic harmonic RMS value $\mathrm{T}_{\mathrm{h} 2}$ and the average value $\mathrm{T}_{\mathrm{av} 2}$ ), in the whole speed range. The main harmonics of Table II are considered.

\section{REFERENCES}

[1] A. Di Gerlando, K. ElShawarby, G. M. Foglia, R. Perini, "DC side current and torque ripples reduction in multi modular PMSG for wind applications," XXII Intern. Conf. on Electrical Machines (ICEM), Lausanne, 2016, pp. 252-258.

[2] A. Di Gerlando; K. ElShawarby; M. Iacchetti, G. M. Foglia; R. Perini: "DC Current and Torque Ripple Mitigation in Modular PMSGs Drives for Multi-MW WECS with Linear PWM Inverter Modulation,' submitted to XXIII Int. Conf. on Electrical Machines (ICEM), 2018.

[3] R. Teodorescu, M. Liserre, P. Rodriguez, "Grid converters for photovoltaic and wind power systems", John Wiley \& Sons Ltd, 2011.

[4] M. Chinchilla, S. Arnaltes and J. C. Burgos, "Control of PM generators applied to variable-speed wind-energy systems connected to the grid," IEEE Trans. on Energy Conv., v. 21, no. 1, pp. 130-135, March 2006.

[5] Y. Errami, M. Maaroufi and M. Ouassaid, "Control scheme and Maximum Power Point Tracking of variable speed wind farm based on the PMSG for utility network connection," 2012 IEEE International Conference on Complex Systems (ICCS), Agadir, 2012, pp. 1-6.

[6] Y. Errami, M. Hilal, M. Benchagra, M. Maaroufi and M. Ouassaid, "Nonlinear control of MPPT and grid connected for wind power generation systems based on the PMSG," 2012 International Conference on Multimedia Computing and Systems, Tangier, 2012, pp. 1055-1060. doi: 10.1109/ICMCS.2012.6320137.

[7] P. Gajewski and K. Pieńkowski, "Analysis of Sliding Mode Control of variable speed wind turbine system with PMSG," 2017 International Symposium on Electrical Machines (SME), Naleczow, 2017, pp. 1-6.

[8] N. Freire, J. Estima, A. Cardosa, "A comparative analysis of PMSG drives based on vector control and direct control techniques for wind turbine applications," Przeglad Elektrot. R.88, n. 1, pp. 184-187, 2012.

[9] P. Gajewski, K. Pieńkowski, "Direct Torque Control and Direct Power Control of wind turbine system with PMSG", Przegląd Elektrotechniczny R.92, no. 10, pp. 249-253, 2016.

[10] J.S. Thongam, M. Tarbouchi, R. Beguenane, A.F. Okou, A. Merabet, P. Bouchard, "A power maximization controller for PMSG wind energy conversion systems", Journal of Electrical and Control Engineering, vol. 3, pp. 1-8, 2013.

[11] A. H. Rajaei, M. Mohamadian and A. Yazdian, "Carrier-based PWM and space vector modulation of three single phase PFC rectifier as generator-side converter of PM WECS," 2012 IEEE Industry Applications Society Annual Meeting, Las Vegas, NV, 2012, pp. 1-8.

[12] M. M. N. Amin and O. A. Mohammed, "DC-Bus Voltage Control Technique for Parallel-Integrated Permanent Magnet Wind Generation Systems," in IEEE Transactions on Energy Conversion, vol. 26, no. 4, pp. 1140-1150, Dec. 2011. doi: 10.1109/TEC.2011.2163409

[13] K. H. Kim, Y. C. Jeung, D. C. Lee and H. G. Kim, "Robust control of PMSG wind turbine systems with back-to-back PWM converters," The 2nd International Symposium on Power Electronics for Distributed Generation Systems, Hefei, China, 2010, pp. 433-437. doi: 10.1109/PEDG.2010.5545751.

[14] D. G. Holmes, T. A. Lipo, "Pulse Width Modulation for Power Converters: Principles and Practice", October 2003, Wiley-IEEE Press, ISBN: 978-0-471-20814-3.

[15] K. Elshawarby, "Sequentially controlled two level inverters of multi modular permanent magnet machines for wind energy systems", Master Degree Thesis, April 27th 2016, Politecnico di Milano, Italy.

\section{BIOGRAPHIES}

Antonino Di Gerlando received his MS degree in electrical engineering from the Politecnico di Milano, Italy, in 1981. Currently, he is a Full Professor at the Department of Energy at Politecnico di Milano. Fields of interest: design and modeling of electrical machines, converters and drive systems. He is a senior member of IEEE and a member of the Italian Association of the Electric and Electronic Engineers (AEI).

Khaled EIShawarby received his MS degree in electrical engineering at Politecnico di Milano, Milano, Italy 2016. Currently, he is a PhD student in Electrical Engineering at Politecnico di Milano, Italy. His interests are in power electronics and electrical machines.

Giovanni Maria Foglia received his MS degree and the $\mathrm{PhD}$ in electrical engineering at Politecnico di Milano, Milano, Italy, in 1997 and 2000.

Currently, he is an Assistant Professor at the Department of Energy at Politecnico di Milano, and his main field of interest is the analysis and design of PM electrical machines.

Roberto Perini (M'10) received his MS degree and the $\mathrm{PhD}$ in electrical engineering from the Politecnico di Milano, Milano, Italy.

Currently, he is an Associate Professor at the Department of Energy at Politecnico di Milano. His interests are in the design and modeling of electrical machines and power electronics. 